\title{
Coupled Locality Preserving Projections for Cross-view Gait Recognition
}

\author{
Wanjiang $\mathrm{Xu}^{\mathrm{a}, \mathrm{b}}$, Can $\mathrm{Luo}^{\mathrm{a}}$, Aiming Ji ${ }^{\mathrm{a}}$, Canyan $\mathrm{Zhu}^{\mathrm{a}, *}$ \\ ${ }^{a}$ Institute of Intelligent Structure and System, soochow university, Suzhou, China 215006 \\ ${ }^{b}$ Yancheng Teachers University, Yancheng, China 224002
}

\begin{abstract}
Existing methods for gait recognition mainly depend on the appearance of human. Their performances are greatly affected by changes of viewing angle. To achieve higher correct classification rates for cross-view gait recognition, we develop a coupled locality preserving projections (CLPP) method in this paper. It learns coupled projection matrices to project cross-view features into a unified subspace while preserving the essential manifold structure. In the projected subspace, cross-view gait features can be matched directly. By the virtue of structure information, the learnt subspace is more robust to the view change. Experiments based on CASIA and USF gait databases are conducted to verify the efficiency of our approach.
\end{abstract}

Keywords: gait recognition, unified subspace, coupled locality preserving projections

\section{Introduction}

Gait recognition aims to identify humans at a distance by inspecting their walking manners. It has a wide range of application for visual surveillance in public areas. However, in such environments there are various factors significantly affecting the performance of gait recognition [1]. One of the most challenging factors is the view change [2].

A variety of approaches have been proposed to tackle the view issue. These approaches fall into three categories: $3 \mathrm{D}$ gait model reconstruction based approach $[3,4,5]$, view-invariant approach $[6,7,8]$, and learning mapping or projection relationships of gait across views approach [9, 10,11,12]. The third category is further divided into two types: view transformation-based and subspace-based approaches. Among these methods, subspace-based approaches, with the advantage of high efficiency and convenience in application, attract a lot of attentions in recent years. For cross-view gait recognition, CCA [12] is one of the most popular approaches, which projects gait features from different views into two subspaces such that they have the largest correlation. And gait features are matched by CCA correlation strength directly. However, CCA needs exactly same amount of samples under different views in training process, which is impractical in real world.

Coupled metric learning $(\mathrm{CML})[13,14]$ is another typical method. It seeks a common subspace where features of cross-view gait could be measured. However, existing methods based on CML do not take into account each collection's own manifold structure while constructing the relationship between different views. For example, in training process, the originally neighboring points in set $X$ or $Y$ may be apart after being projected; then in testing process, the points may be projected in unexpected direction, since the original local relationship in each data set was not preserved. As shown in Figure 1, the distance of projected points $z_{4}$ and $z_{5}$ from different classes may be closer than that of $z_{2}$ and $z_{5}$ from the same class.

In this work, we manage to seek a coupled projections approach which is more suitable for classification of crossview gait data. On basis of CML, we attempt to preserve the manifold structure of gait features in each view while simultaneously maximize the relevance of gait features from two different views. The coupled projection matrices

\footnotetext{
${ }^{*}$ Corresponding author

Email addresses: xuwanjiang0821@163.com (Wanjiang Xu), 20134046005@stu.suda.edu.cn (Can Luo), jiaiming@suda.edu.cn (Aiming Ji), qi wuzhu@suda. edu.cn (Canyan Zhu)
} 


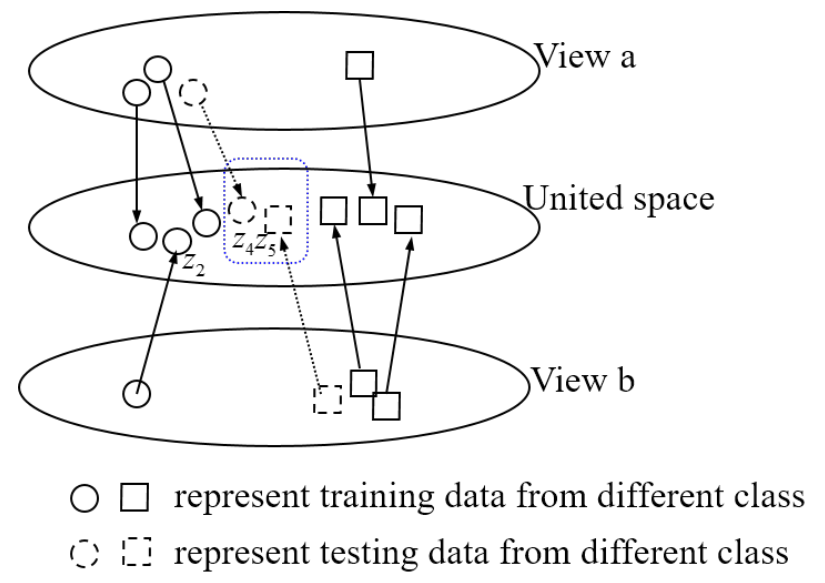

Figure 1: Neighboring nodes in same view scattered after mapped into a unified space.

are firstly learnt to map original feature spaces into a unified subspace. Then in the learnt subspace, we measure the projected features obtained from gallery and probe gait sequences in Euclidean distance.

The key contributions of this paper can be summarized as follows.

- A novel coupled projections algorithm named Coupled Locality Preserving Projections (CLPP) is proposed, which can project sample points from two different viewing spaces into a unified subspace while preserving the manifold structure of intra-view and correlation of inter-view.

- This paper proposed a practical approach calculating the inter-view similarity matrix by searching the maxweighted path, which avoid being simply substituted by intra-view similarity matrix.

- To further evaluate effectiveness of the proposed method, extensive experiments are carried out for cross-view gait recognition under various scenarios which are quite common in real world.

The rest of this paper is organized as follows. Related works are discussed in section 2. In section 3, we briefly review the CML based approach and then introduce our proposed approach formally. Feature presentation, extraction and classification of gait data are described in section 4. Experimental results are shown in section 5 and conclusions are drawn in section 6 .

\section{Related Work}

\subsection{Cross-View Gait Recognition}

Current research on gait recognition across various viewing angles falls into three categories. The approaches in the first category rely on the reconstructed 3D gait model $[3,4,5,15,16]$. Bodor et al. [3] computed a 3D visual hull model using gait silhouettes from multiple views. Taking advantage of 3D visual hull model, they could reconstruct gait features under any required view. Zhao et al. [15] reconstructed a 3D human skeleton model by multiple cameras. From 3D models, the lengths of key segments were extracted as static features and motion trajectories of lower limbs were extracted as dynamic features. Linear time normalization was exploited for matching and recognition. Iwashita et al. [16] created a spatio-temporal 3D gait database directly to synthesize gait sequence at each viewpoint. By comparing the gait features with those in the database, the person was identified and his walking direction was estimated. All these approaches achieved high accuracy in their experiments. However, on account of expensive computation and complex camera calibration, this family of approaches is only suitable for a fully controlled and cooperative multi-camera environment such as a bio-metric tunnel [5].

The second category $[6,7,8,17]$ is to extract view-invariant gait features. Jean et al. [6] proposed a method to compute view normalized feet and head $2 \mathrm{D}$ trajectories as view-invariant gait features. By homography based 
transformation, the trajectories were normalized in a lateral viewpoint for gait recognition. Goffredo et al. [8] proposed self calibrating view-invariant gait recognition based on human joint's position estimation and viewpoint rectification. Angular measurements and trunk spatial displacement were derived from the rectified limbs' poses and used as a viewinvariant gait feature. Kusakunniran et al. [17] transformed gait silhouettes from arbitrary view onto the canonical view using domain transformation. Procrustes mean shape was extracted as feature to measure gait similarity. When difference between two views was large, these methods can still perform efficiently. However, these methods in the category are not applicable for front view because the gait feature from front view could not be transformed onto side view.

The third category learns mapping or projection relationship of gaits across views through a training process. The learnt relationships will normalize gait features from different views into shared or associated subspace(s) before gait similarity is measured. Different from the first category, the third category needs only one simple camera. The relationship between gait data from different views is established through the learning process. Compared with the second category, the third category is more efficient and stable. In addition, there is no limitation to the view point. Recent researches in the third category mainly rely on view transform model (VTM) $[9,10,11,18,19]$ and subspace learning $[12,20,21,22,23,24]$. VTM was introduced [18] to transform gait features from one view into another view. Frequency-domain gait features from different views were used to form a large matrix. Then the matrix was factorized by adopting singular value decomposition (SVD) to establish the VTM. Kusakunniran et al. [10] created a VTM using support vector regression based on local dynamic feature extraction. Sparse regression-based VTM was also proposed [11] to obtain stable model for VTM construction. Lately, Muramatsu et al. [19] developed an arbitrary view transform model (AVTM) by combining aspects of both first and third categories. 3D gait visual hulls were established and used to generate training gait sequences under any required views. Then VTM was constructed to transform features.

Recently, subspace learning based approaches have been adopted to transform the gait features obtained from various viewing spaces into a shared feature space. Bashir et al. [12], using canonical correlation analysis (CCA), learnt maximally correlated feature subspaces and employed correlation strength to measure gait similarity. A viewinvariant discriminative projection (ViDP) method was introduced [22] to learn a low dimensional feature subspace. It was implemented by iteratively learning the low dimensional geometry and finding the optimal projection according to the geometry. $\mathrm{Hu}$ [23] proposed an uncorrelated multilinear sparse local discriminant canonical correlation analysis (UMSLDCCA) approach to model the correlations of gait features from different viewing angles. A tensor-to-vector projection (TVP) was adopted to extract gait features for measuring similarity. Xing et al. [24] proposed complete CCA (C3A) to overcome the singular problem of covariance matrix and alleviate the computational burden of high dimensional matrix for typical gait image data. Compared with VTM, subspace learning based methods can cope with feature mismatch across views and are more robust against feature noise.

\subsection{Subspace learning}

Subspace learning sheds light on various tasks in computer vision and multimedia. It projects the original feature space(s) to a new subspace, wherein specific statistical properties can be well preserved. The representative approaches include principal component analysis (PCA) [25], Linear Discriminant Analysis (LDA) [26] and Locality preserving projections (LPP) [27]. PCA is an unsupervised dimension reduction algorithm, while LDA is a supervised one. LPP can either be supervised or unsupervised by changing similarity matrix. LPP has been applied in many applications. However, it does not work well when data are from two different sets (such as gait data from two different views). The main reason is that the similarity matrix could not be constructed well between two different sets.

CCA is probably the most popular two-view subspace learning method due to its wide-spread use in cross-view biometric recognition [28, 29], cross-media retrieval [30, 31] and some other problems [32]. Li et al. [29] applied CCA to face recognition based on non-corresponding region matching. An et al. [32] used regularized CCA (RCCA) in conjunction with a reference set for person re-identification. Luo et al. [30] developed tensor CCA (TCCA) by analyzing the high-order covariance tensor over the data from all views. A more reliable common subspace shared by all features could be obtained.

Besides CCA, there are some other methods for two-view subspace learning. Sharma and Jacobs [33] used partial least squares (PLS) to linearly map images from different modalities to a common linear subspace in which they are highly correlated. Tenenbaum and Freeman [28] proposed a bilinear model (BLM) to derive a unified space for crossmodal face recognition. Li et al. [13] proposed coupled metric learning (CML) approach to learn a common space in 
which degraded face recognition was performed. Jacobs et al. [33] extended Linear discriminant analysis (LDA) and marginal fisher analysis (MFA) to their multiview counterparts and applied them to deal with the cross-media retrieval problem.

Lately, inspired by the ability of deep learning models to learn nonlinear representations, a topic of interest has been to design cross-view subspace learning methods via deep structures. Andrew et al. [34] proposed the deep canonical correlation analysis (DCCA) method, which is nonlinear extension of CCA. In brief, DCCA built two deep networks for two different views, and the representations of the highest layer of the networks were constrained to be correlated. Wang et al. [35] introduced the deeply coupled auto-encoder networks (DCAN) method to effectively employ the label information of the training data. Similar to DCCA, DCAN constructed two auto-encoder networks, which were discriminatively coupled with each other in every corresponding layer. Benefit from deep structure, the subspace learnt from DCCA or DCAN has more discrimination capability.

\section{Coupled locality preserving projections}

\subsection{Coupled metric learning}

The approaches [21, 14] based on coupled metric learning attempted to project the sample features (vectors) from two different spaces into a unified space by coupled mapping : one for $\mathbf{x}_{i} \in X: \mathfrak{R}^{m_{x}} \rightarrow \mathfrak{R}^{d}$, and the other for $\mathbf{y}_{j} \in Y: \mathfrak{R}^{m_{y}} \rightarrow \mathfrak{R}^{d}$. Their distance was measured in the new space. The metric can be expressed as follows:

$$
d_{C}\left(\mathbf{x}_{i}, \mathbf{y}_{j}\right)=d\left(f_{x}\left(\mathbf{x}_{i}\right), f_{y}\left(\mathbf{y}_{j}\right)\right)=\left\|\mathbf{P}_{C}^{T} f_{x}\left(\mathbf{x}_{i}\right)-\mathbf{P}_{C} f_{y}\left(\mathbf{y}_{j}\right)\right\|_{2},
$$

where $f_{x}(\cdot)$ and $f_{y}(\cdot)$ were projection functions of $X$ and $Y$ respectively. $\mathbf{P}_{\mathbf{c}}$ was a linear transformation to be learnt. Further, let $f_{x}\left(\mathbf{x}_{i}\right)=\mathbf{P}_{x}^{T} \mathbf{x}_{i}$ and $f_{y}\left(\mathbf{y}_{i}\right)=\mathbf{P}_{y}^{T} \mathbf{y}_{i}$, then we can get $\mathbf{W}_{x}=\mathbf{P}_{x} \mathbf{P}_{C}$ (with size of $m_{x} \times d$ ) and $\mathbf{W}_{y}=\mathbf{P}_{y} \mathbf{P}_{C}$ (with size of $\left.m_{y} \times d\right)$. Finally the metric became

$$
d_{C}\left(\mathbf{x}_{i}, \mathbf{y}_{j}\right)=d\left(f_{x}\left(\mathbf{x}_{i}\right), f_{y}\left(\mathbf{y}_{i}\right)\right)=\left\|\mathbf{W}_{x}^{T} \mathbf{x}_{i}-\mathbf{W}_{y}^{T} \mathbf{y}_{j}\right\|_{2} .
$$

The coupled metric learning was formulated by following objective function:

$$
J\left(\mathbf{W}_{x}, \mathbf{W}_{y}\right)=\sum_{i, j}\left\|\mathbf{W}_{x}^{T} \mathbf{x}_{i}-\mathbf{W}_{y}^{T} \mathbf{y}_{j}\right\|^{2} .
$$

Ben [21] improved CML by preserving similarity relationship. Their objection was to minimize following formulation:

$$
J\left(\mathbf{W}_{x}, \mathbf{W}_{y}\right)=\sum_{i}^{n_{x}} \sum_{j}^{n_{y}}\left(\mathbf{W}_{x}^{T} \mathbf{x}_{i}-\mathbf{W}_{y}^{T} \mathbf{y}_{j}\right)^{2} s_{i j}^{x y} .
$$

where $n_{x}$ and $n_{y}$ indicated the number of samples from X and Y. $s_{i j}^{x y}$ was similarity metric between sample $\mathbf{x}_{i}$ and $\mathbf{y}_{j}$. However, the value $s_{i j}^{x y}$ was hard to be achieved from cross-view collections $\mathrm{X}$ and $\mathrm{Y}$, because they had different mean center and variance and they had different manifold. In their application [21], $s_{i j}^{x y}$ was replaced by similarity value $s_{i j}^{x}$ between $\mathbf{x}_{i}$ and $\mathbf{x}_{j}$ (corresponding to $\mathbf{y}_{j}$ ) in same collection X. Besides, the objective functions of existing CML based approaches only included the inter-view relation between $\mathbf{x}_{i}$ and $\mathbf{x}_{j}$, without considering intra-view relation such as relationships between $\mathbf{y}_{i}$ and $\mathbf{x}_{j}$ from one collection. Consequently, their algorithms were severely limited in many applications.

\subsection{Proposed method}

For a cross-view problem, we expect to find two mapping matrices, which could project features from either space of two different views into a unified subspace then they can be measured directly. In order to avoid the limitations of existing CML based methods, the projected common subspace should not only preserve the similarity relationship between two different view data, but also keep the essential manifold structure of the respective collection. On basis of formula (4), our objective function is as follows:

$$
J\left(\mathbf{W}_{x}, \mathbf{W}_{y}\right)=\sum_{i, j}\left(\mathbf{W}_{x}^{T} \mathbf{x}_{i}-\mathbf{W}_{y}^{T} \mathbf{y}_{j}\right)^{2} s_{i j}^{x y}+\frac{1}{2} \sum_{i, k}\left(\mathbf{W}_{x}^{T} \mathbf{x}_{i}-\mathbf{W}_{x}^{T} \mathbf{x}_{k}\right)^{2} s_{i k}^{x}+\frac{1}{2} \sum_{j, l}\left(\mathbf{W}_{y}^{T} \mathbf{y}_{j}-\mathbf{W}_{y}^{T} \mathbf{y}_{l}\right)^{2} s_{j l}^{y} .
$$


where $i=1,2, \ldots, n_{x}, k=1,2, \ldots, n_{x}, j=1,2, \ldots, n_{y}$ and $l=1,2, \ldots, n_{y}$. Similarity metric (also called penalty weight) is used to represent neighboring relationships: $s_{i j}^{x y}$ is similarity metric between $\mathbf{x}_{i}$ and $\mathbf{y}_{j}$; $s_{i k}^{x}$ is similarity metric between $\mathbf{x}_{i}$ and $\mathbf{x}_{k} ; s_{j l}^{y}$ is similarity metric between $\mathbf{y}_{j}$ and $\mathbf{y}_{l}$. Let $\mathbf{X}=\left[\mathbf{x}_{1}, \mathbf{x}_{2}, \ldots, \mathbf{x}_{n_{x}}\right]$ and $\mathbf{Y}=\left[\mathbf{y}_{1}, \mathbf{y}_{2}, \ldots, \mathbf{y}_{n_{y}}\right]$, the formula (5) could be written as follows (Detailed mathematical derivation is presented in Appendix A) :

$$
J\left(\mathbf{W}_{\mathbf{x}}, \mathbf{W}_{\mathbf{y}}\right)=\operatorname{tr}\left(\mathbf{W}^{T} \mathbf{Z}(\mathbf{D}-\mathbf{S}) \mathbf{Z}^{\mathbf{T}} \mathbf{W}\right)=\operatorname{tr}\left(\mathbf{W}^{\mathbf{T}} \mathbf{Z} \mathbf{L} \mathbf{Z}^{\mathbf{T}} \mathbf{W}\right) .
$$

where $\mathbf{Z}=\left[\begin{array}{cc}\mathbf{X} & \\ & \mathbf{Y}\end{array}\right], \mathbf{W}=\left[\begin{array}{c}\mathbf{W}_{x} \\ \mathbf{W}_{y}\end{array}\right], \mathbf{S}=\left[\begin{array}{cc}\mathbf{S}_{x} & \mathbf{S}_{x y} \\ \mathbf{S}_{x y}^{T} & \mathbf{S}_{y}\end{array}\right]$, D is a diagonal matrix as $D_{i i}=\sum_{j} S_{j i}$ and $\mathbf{L}=\mathbf{D}-\mathbf{S}$ is the Laplacian matrix. $\mathbf{W}_{x}$ and $\mathbf{W}_{y}$ are the project matrices of $\mathbf{X}$ and $\mathbf{Y} ; \mathbf{S}_{x}$ and $\mathbf{S}_{y}$ are the similarity matrix of $\mathbf{X}$ and $\mathbf{Y}$, and they are symmetric; $\mathbf{S}_{x y}$ is the similarity matrix between $\mathbf{X}$ and $\mathbf{Y} . s_{i k}^{x}$, $s_{j l}^{y}$ and $s_{i j}^{x y}$ are elements of $\mathbf{S}_{x}, \mathbf{S}_{y}$ and $\mathbf{S}_{x y}$ respectively.

Equation (6) has a similar objective function as LPP. In this paper, it is called Coupled Locality Preserving Projections (CLPP), because it learns coupled projections for two different sets. Finally, we solve the optimization problem by minimizing:

$$
J\left(\mathbf{W}_{x}, \mathbf{W}_{y}\right)=\operatorname{tr}\left(\mathbf{W}^{\mathbf{T}} \mathbf{Z} \mathbf{L} \mathbf{Z}^{\mathbf{T}} \mathbf{W}\right) \quad \text { S.t. } \quad \mathbf{W}^{T} \mathbf{Z} \mathbf{D Z} \mathbf{Z}^{\mathbf{T}} \mathbf{W}=\mathbf{I} .
$$

where $\mathbf{I}$ is the identity matrix with size of $d \times d$. The solution to make Formula (7) minimized is obtained by a generalized eigen-decomposition of $\mathbf{Z L Z} \mathbf{Z}^{T} \mathbf{w}=\lambda \mathbf{Z D Z} \mathbf{Z}^{T} \mathbf{w} . \mathbf{W}$ is constructed by the eigenvectors $\left[\mathbf{w}_{1}, \mathbf{w}_{2}, \ldots, \mathbf{w}_{d}\right]$ according to the $d$ smallest eigen-values. The first $m_{x}$ rows of $\mathbf{W}$ corresponds to $\mathbf{W}_{\mathbf{x}}$ and the rest rows corresponds to $\mathbf{W}_{\mathbf{y}}$.

Our approach CLPP is to find an embedding that preserves the similarity relationship, while maintaining the manifold structure of each collection. To achieve this, our approach relies greatly on similarity matrices.

\subsection{Similarity matrices for coupled locality preserving projections}

$X$ and $Y$ are collections from different views. $\mathbf{S}_{\mathbf{x}}\left(\right.$ or $\mathbf{S}_{\mathbf{y}}$ ) is the similarity matrix in one collection $X($ or $Y$ ). We define $\mathbf{S}_{\mathbf{x}}$ of which element is $s_{i j}^{x}$ as follows:

$$
s_{i j}^{x}=\left\{\begin{array}{cc}
\exp \left(-\frac{\left\|x_{i}-x_{j}\right\|^{2}}{t}\right) & \text { if } x_{j} \in K N N\left(x_{i}\right) \\
0 & \text { otherwise }
\end{array}\right.
$$

Each element $s_{i j}^{y}$ of similarity matrix $\mathbf{S}_{\mathbf{y}}$ is defined in the same way as $s_{i j}^{x}$. Unlike previous two intra-view similarity matrix $\mathbf{S}_{\mathbf{x}}$ and $\mathbf{S}_{\mathbf{y}}, \mathbf{S}_{\mathbf{x y}}$ is the inter-view similarity matrix. Since there are no similarity comparable between $\mathbf{X}$ and $\mathbf{Y}$ in Euclidean distance, the only relevant information is their labels. For two sample points $\mathbf{x}_{k} \in \mathbf{X}\left(k=1,2, \ldots, n_{x}\right)$ and $\mathbf{y}_{l} \in \mathbf{Y}\left(l=1,2, \ldots, n_{y}\right)$, we construct their relationships by whether they have the same class label. Their relation value $c_{k l}^{x y}$ can be defined as follows:

$$
c_{k l}^{x y}=\left\{\begin{array}{cc}
1 & \text { if label }\left(x_{k}\right)=\operatorname{label}\left(y_{l}\right) \\
0 & \text { otherwise }
\end{array}\right.
$$

Then, we can construct final inter-view similarity matrix $\mathbf{S}_{\mathbf{x y}}$ between $\mathbf{X}$ and $\mathbf{Y}$. Each element $s_{i j}^{x y}$ can be calculated as follows:

$$
s_{i j}^{x y}=\max _{k, l} s_{i k}^{x} \cdot c_{k l}^{x y} \cdot s_{l j}^{y}
$$

For example, as shown in Figure 2, there are three paths from $x_{1}$ to $y_{5}$. It means that we can get $s_{1,5}^{x y}$ from the maximum of $s_{1,2}^{x} \cdot c_{2,2}^{x y} \cdot s_{2,5}^{y}, s_{1,3}^{x} \cdot c_{3,3}^{x y} \cdot s_{3,5}^{y}$ and $s_{1,4}^{x} \cdot c_{4,4}^{x y} \cdot s_{4,5}^{y}$. 


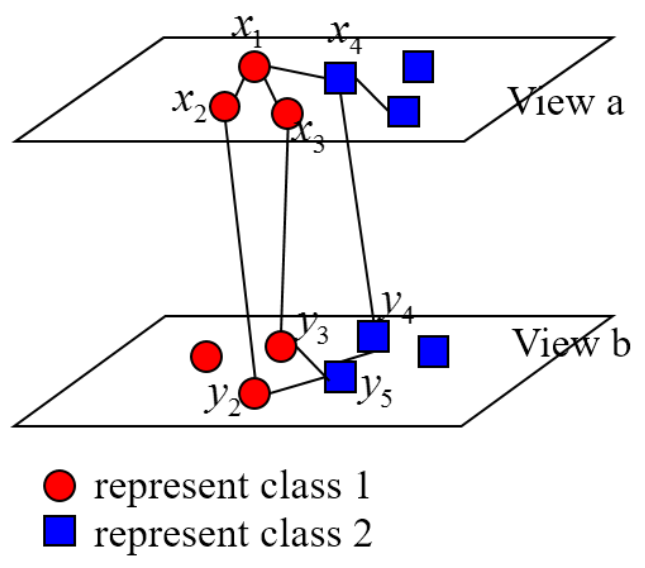

Figure 2: Similarity computation for cross-view data.

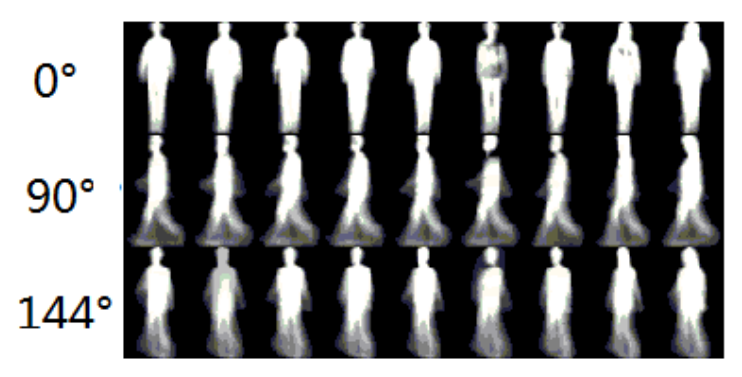

Figure 3: GEIs under different views. Each row shows GEIs of different person under same view. Different rows correspond to different views.

\section{Cross-view gait recognition}

\subsection{Gait presentation}

In this paper, we use well known Gait Energy Image (GEI) [36, 22] as gait feature. GEI is constructed from a sequence of aligned gait images in a window of complete walking cycle(s) because gait is a kind of periodic action. It contains rich gait information preserving a spatial representation of human shape. The rich content of GEI will provide a substantial relationship across different views which is naturally bridged by spatial information. As shown in Figure 3, examples of GEI are from different subjects under various views on the CASIA gait database B. For each viewing angle, various samples look similarity, which may form a Riemann manifold independently. However, GEIs of the same person vary greatly under different views.

\subsection{Feature extraction}

Let each GEI be vectorized firstly, then two given training GEI sets from different views are represented in the form of two matrices $\mathbf{X}_{\text {train }}$ and $\mathbf{Y}_{\text {train }}$ with the size of $m_{x} \times n_{x}$ and $m_{y} \times n_{y}$ respectively. We use our method to obtain coupled mapping matrices $\mathbf{W}_{x} \in \mathfrak{R}^{m_{x} \times d}$ and $\mathbf{W}_{y} \in \mathfrak{R}^{m_{y} \times d}$ in the training process. They are used to project gait feature into a unified subspace.

Therefore, in the testing phase, $\mathbf{x} \in \mathfrak{R}^{m_{x}}$ and $\mathbf{y} \in \mathfrak{R}^{m_{y}}$ can be used to projected into learnt subspace as $\mathbf{z}_{x}=\mathbf{W}_{x}^{T} \cdot \mathbf{x}$ and $\mathbf{z}_{y}=\mathbf{W}_{y}^{T} \cdot \mathbf{y}\left(\mathbf{z}_{x} \in \mathfrak{R}^{d}\right.$ and $\left.\mathbf{z}_{y} \in \mathfrak{R}^{d}\right)$ respectively.

\subsection{Classification}

To perform cross-view recognition, we have gait templates of subjects in one view as our gallery data. Any probe gait sequence in another view can now be recognized as the same subject in the gallery. 
The identity is estimated by the nearest neighbor classifier, which can be written as

$$
\underset{i \in 1,2, l l}{\arg \min }\left\|\mathbf{W}_{x}^{T} \mathbf{x}_{i}-\mathbf{W}_{y}^{T} \mathbf{y}_{j}\right\|^{2},
$$

where $l$ is the amount of gallery samples.

\section{Experiment and analysis}

In this paper, the proposed coupled locality preserving projections approach is tested on the two most widely-used gait database: CASIA(B) and USF. The CASIA(B) gait database is firstly used to discuss the parameter selection for constructing the similarity matrices. Then, the proposed approach is used to evaluate the performance across different views. The two database are both divided into two sets: first half of samples for training and rest for testing. In the experiments, one of the gait recognition problem is confronted with the difficulty that the matrix is sometimes singular. To overcome this problem, PCA is used to reduce the dimensions of GEI features in our experiments.

\subsection{Experiments on the CASIA gait database}

The CASIA gait database B [2] is applied in our experiments. This database directly supports the paper of gait recognition under view change, which contains 124 subjects from 11 views $\left(0^{\circ}, 18^{\circ}, \ldots, 180^{\circ}\right)$. There are six gait sequences for each subject from each view. The first 62 subjects six sequences of each view are used in training process while the latter 62 subjects sequences are evaluated in testing process.

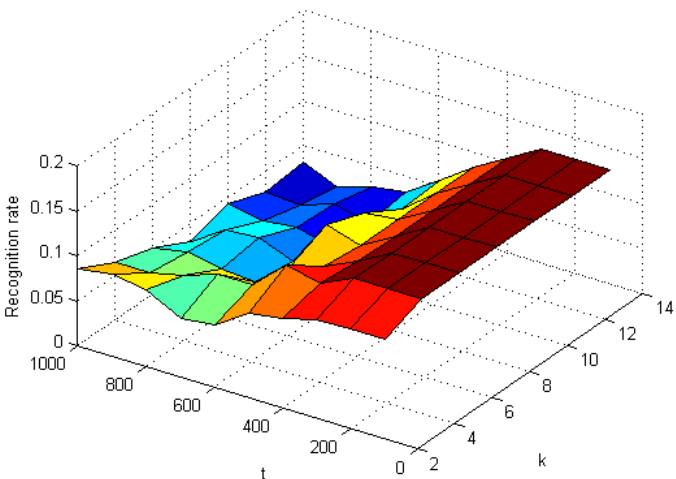

(a) Recognition rates under view $0^{\circ}$ and $90^{\circ}$.

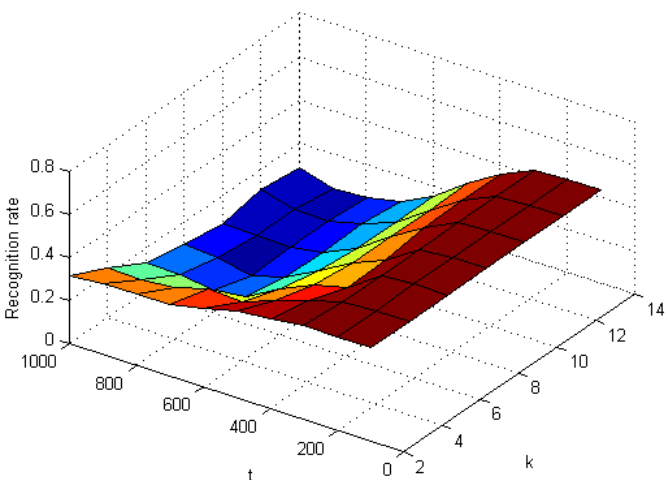

(b) Recognition rates under view $54^{\circ}$ and $90^{\circ}$.

Figure 4: Recognition rates over the variations of number $\mathrm{k}$ of nearest neighbors and scaling factor $\mathrm{t}$.

As shown in Figure 4, the correct recognition rates with varying number $\mathrm{k}$ of nearest neighbors and scaling factor $\mathrm{t}$ are different. In cross-validation experiment on training set, we found that the top average recognition rates can be reached under the condition of $t \in[100,300]$ and $k \in[4,14]$. Therefore, in follow-up experiment, $t$ is set to 200 and $k$ is set to 4 .

A comprehensive experiment is conducted to compare the performance of the proposed method with that of LPP [27], Bashir's method [12] (CCA), Li's method [13] (CML) and Ben's method [21]. In our experiments, for a fair comparison, we have carried out experiments based on these methods and the proposed method on the same training and testing data sets.

Recognition rates of our method and other methods are summarized in Table 1. It is clearly seen that cross-view gait recognition between two closer views achieves better performance because of more shared gait information. The performance of classical LPP is worst. This is because it can not preserve real manifold structure for cross-view features. 
Table 1: Comparisons on cross-view gait recognition rate (\%) using different methods.

\begin{tabular}{|c|c|c|c|c|c|c|c|c|c|c|c|}
\hline Gallery & \multicolumn{11}{|c|}{$0^{\circ}$} \\
\hline Probe & $18^{\circ}$ & $36^{\circ}$ & $54^{\circ}$ & $72^{\circ}$ & $90^{\circ}$ & $108^{\circ}$ & $126^{\circ}$ & $144^{\circ}$ & $162^{\circ}$ & $180^{\circ}$ & mean \\
\hline LPP & 51 & 9 & 1 & 1 & 2 & 2 & 2 & 5 & 4 & 19 & 10 \\
\hline $\mathrm{CCA}[12]$ & 59 & 17 & 3 & 6 & 1 & 2 & 6 & 12 & 27 & 65 & 27 \\
\hline CML[13] & 27 & 10 & 5 & 5 & 1 & 3 & 6 & 5 & 5 & 30 & 18 \\
\hline Ben's method[21] & 49 & 14 & 2 & 5 & 0 & 1 & 3 & 3 & 18 & 54 & 23 \\
\hline Our method & 54 & 18 & 6 & 6 & 9 & 11 & 9 & 16 & 34 & 62 & 29 \\
\hline Gallery & \multicolumn{11}{|c|}{$54^{\circ}$} \\
\hline Probe & $0^{\circ}$ & $18^{\circ}$ & $36^{\circ}$ & $72^{\circ}$ & $90^{\circ}$ & $108^{\circ}$ & $126^{\circ}$ & $144^{\circ}$ & $162^{\circ}$ & $180^{\circ}$ & mean \\
\hline LPP & 2 & 22 & 4 & 5 & 16 & 3 & 4 & 4 & 4 & 6 & 7 \\
\hline $\mathrm{CCA}[12]$ & 5 & 40 & 85 & 94 & 44 & 21 & 25 & 16 & 9 & 9 & 40 \\
\hline CML[13] & 4 & 10 & 51 & 42 & 13 & 4 & 5 & 3 & 1 & 6 & 22 \\
\hline Ben's method[21] & 5 & 27 & 73 & 88 & 33 & 20 & 17 & 12 & 7 & 6 & 35 \\
\hline Our method & 15 & 42 & 78 & 85 & 58 & 40 & 43 & 32 & 22 & 16 & 48 \\
\hline Gallery & \multicolumn{11}{|c|}{$90^{\circ}$} \\
\hline Probe & $0^{\circ}$ & $18^{\circ}$ & $36^{\circ}$ & $54^{\circ}$ & $72^{\circ}$ & $108^{\circ}$ & $126^{\circ}$ & $144^{\circ}$ & $162^{\circ}$ & $180^{\circ}$ & mean \\
\hline LPP & 4 & 1 & 9 & 6 & 10 & 22 & 2 & 2 & 2 & 3 & 6 \\
\hline $\mathrm{CCA}[12]$ & 2 & 8 & 16 & 39 & 91 & 88 & 51 & 19 & 10 & 5 & 33 \\
\hline CML[13] & 1 & 8 & 8 & 15 & 65 & 53 & 21 & 7 & 5 & 4 & 19 \\
\hline Ben's method[21] & 1 & 3 & 8 & 25 & 86 & 85 & 35 & 13 & 7 & 3 & 27 \\
\hline Our method & 13 & 13 & 23 & 43 & 85 & 83 & 49 & 25 & 19 & 9 & 36 \\
\hline Gallery & \multicolumn{11}{|c|}{$126^{\circ}$} \\
\hline Probe & $0^{\circ}$ & $18^{\circ}$ & $36^{\circ}$ & $54^{\circ}$ & $72^{\circ}$ & $90^{\circ}$ & $108^{\circ}$ & $144^{\circ}$ & $162^{\circ}$ & $180^{\circ}$ & mean \\
\hline LPP & 2 & 2 & 6 & 2 & 46 & 14 & 31 & 51 & 10 & 7 & 17 \\
\hline $\mathrm{CCA}[12]$ & 9 & 4 & 12 & 22 & 28 & 59 & 94 & 94 & 41 & 14 & 38 \\
\hline CML[13] & 4 & 4 & 5 & 2 & 9 & 17 & 54 & 69 & 8 & 4 & 18 \\
\hline Ben's method[21] & 7 & 3 & 11 & 15 & 16 & 38 & 89 & 91 & 32 & 9 & 31 \\
\hline Our method & 10 & 18 & 25 & 38 & 51 & 59 & 87 & 81 & 44 & 17 & 43 \\
\hline
\end{tabular}

Among the CML-based methods, the proposed method provides the highest accuracy in almost all cases. The original CML [13] perform worst, because it dose not consider any discrimination information. Ben's method [21] achieves competitive recognition rates in some case of relatively small view-difference. However, on account of ignoring the structure information, it is very sensitive to view change. For example, in the case that probe view is $90^{\circ}$ and gallery view is $108^{\circ}$ (18 degree of view change) from Table 1, Ben's method [21] achieves $85 \%$ which is little better than $83 \%$ achieved by the proposed method. When view-difference increase to 36 degree (gallery view is $126^{\circ}$ and probe view is $90^{\circ}$ ), precision of method [21] drops to only $35 \%$ which is less than half of $85 \%$.

From Table 1, it is seen that the proposed method achieves the best average recognition rate. In most cases, the proposed method outperforms other methods. However, in the cases of small view-difference, CCA achieves the best performances. Take probe view under $54^{\circ}$ for example from table 1 . When gallery views are $36^{\circ}$ and $72^{\circ}, \mathrm{CCA}$ achieves $85 \%$ and $94 \%$ which are better than the proposed method. According to our analysis, CCA concentrates on maximizing correlation between gait data from different views. Different from CCA, our method takes into account of both correlation information and manifold structure information. The role of structure information is to weaken the influence of viewing angle change. When difference of viewing angles is small, the manifold structures of gait data from these two views are so similar that the effect of structure factor could be neglected. Therefore, only in the case of small viewing variation, CCA performs little better than the proposed method. In other cases, the proposed method significantly outperforms other methods. For example, when probe view is $54^{\circ}$ and gallery view is $0^{\circ}, 18^{\circ}, 90^{\circ}, 108^{\circ}$, $126^{\circ}, 144^{\circ}, 162^{\circ}$, or $180^{\circ}$, the proposed method achieves better performances than CCA. Particularly, in the cases of large view-difference (gallery view is $108^{\circ}, 126^{\circ}, 144^{\circ}, 162^{\circ}$, or $180^{\circ}$ ), the recognition rate of the proposed method is approximately twice as much as which achieved by CCA.

Moreover, cumulative match score (CMS) curves in Figure 5 are used to further demonstrate the performance of cross-view gait recognition. It is noted that the horizontal axis of Figure 5 is rank (top $n$ matches) and the vertical axis is the recognition rate. In this case, gallery view is $90^{\circ}$ and probe views are $0^{\circ}, 54^{\circ}, 108^{\circ}$, and $144^{\circ}$ respectively. As shown in Figure 5, our proposed method performs best at almost every rank in CMS curves. Even in large view 
Table 2: The performances (\%) on the USF gait database by different methods.

\begin{tabular}{cccccc}
\hline PCA[25] & LPP[27] & CCA[12] & CML[13] & Ben[21] & Our method \\
\hline 70 & 70 & 77 & 77 & 82 & 85 \\
\hline
\end{tabular}

different (probe view is $0^{\circ}$ and gallery view is $90^{\circ}$ ), our method can reach recognition rate of $70 \%$ at rank 20 .

\subsection{Experiments on USF database}

USF database is another widely-used gait database, which was collected outdoors. Many experiments were designed to investigate the effect of five factors on performance, including surface type, shoe-wear type, weight carried, camera angle, and time. Sarkar et al. [1] fixed one gallery set and created twelve probe sets to test performance under different condition. Individuals are unique in the gallery and probe sets, and no common sequences exist between the gallery set and all probe sets.
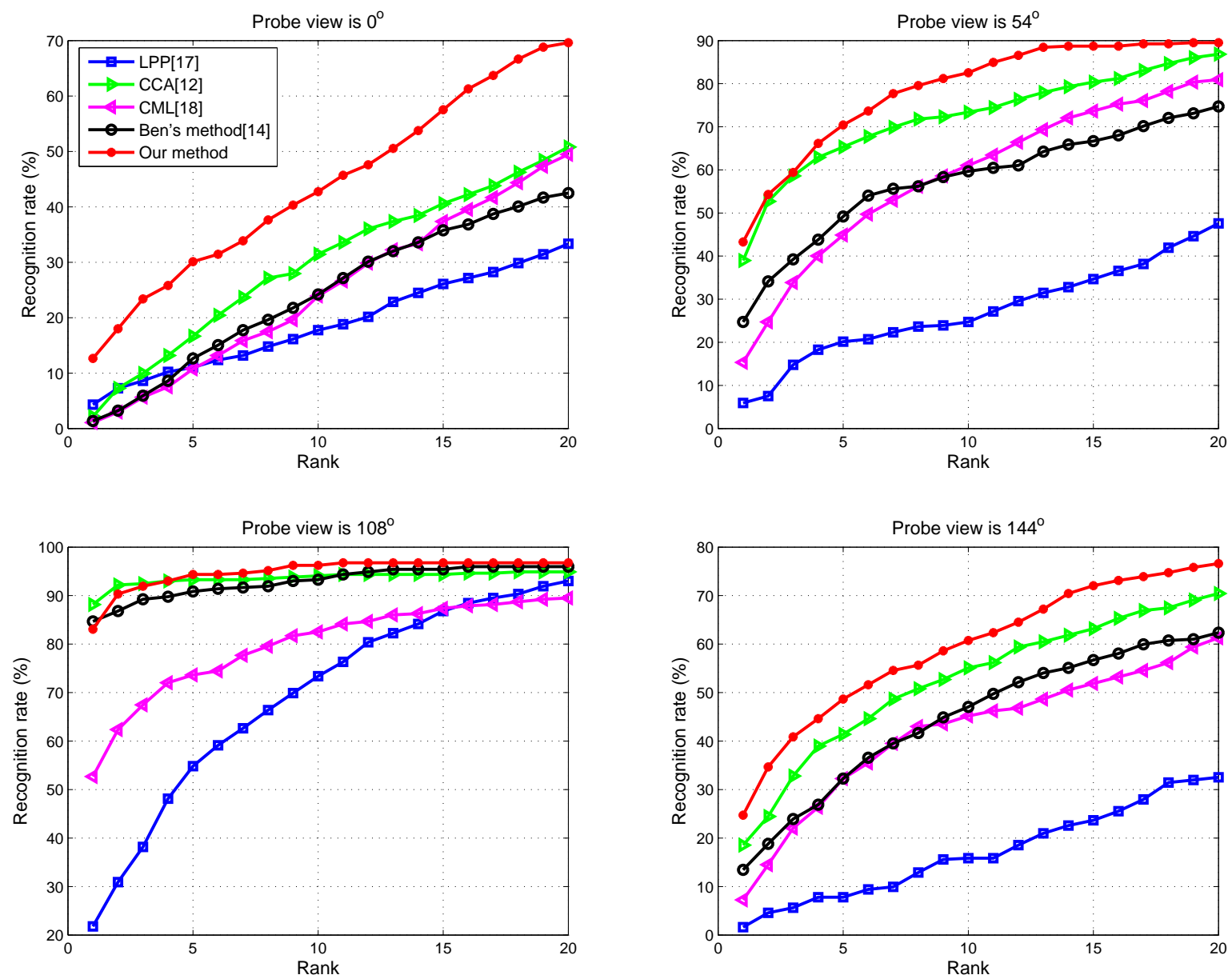

Figure 5: CMS-comparisons on cross-view gait recognition (\%) using different methods. Gallery view is $90^{\circ}$.

Experiment probe A is adopted for our evaluation because it observes view change for gait-based identification, which matches the focus of this paper. In the experiment, two gait sequences were recorded from different cameras $\mathrm{L}$ and $\mathrm{R}$ respectively. The cameras lines of sight are verged at approximately $30^{\circ}$. In our experiment, first 61 subjects are 


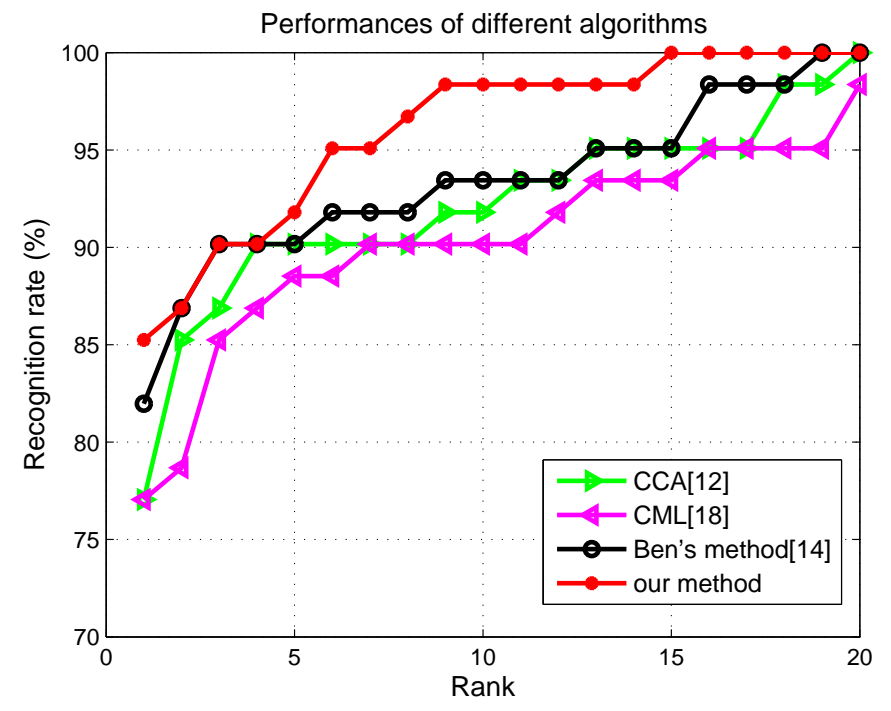

Figure 6: CMS Performances of different methods on USF gait database.

used to train the projection matrices. The remaining 61 subjects are used for evaluation. The performance is shown in Table 2. We can see that the performance of our approach is better than the others. From Table 2, the coupled projections based methods (such as CCA, CML and our method) can reach higher corrected classification rates than single projection method (for example: PCA, LPP) in cross-view gait recognition problem. Among these methods, the proposed method achieves the best recognition rate of $85 \%$ for rank-1.

Further, we compute the accumulative recognition rates for different coupled projections based methods that as shown in Figure 6. It can be seen that our proposed method is a more effective strategy to improve the recognition performance for cross-view gait data. The proposed method outperforms other methods of [12], [21] and [13] from CMS curves.

\section{Conclusion}

In this paper, we proposed a new approach called coupled locality preserving projections (CLPP) to address the gait recognition under view change. In training process, we constructed three similarity matrices: two intra-view similarity matrices for each view and one inter-view similarity matrix. Then, coupled projection matrices for different views were learnt by CLPP. In testing process, we used the projection matrices to project gallery and probe gait data into the learnt subspace and classified probe data in Euclidean distance directly. Experiments on CASIA-B and USF gait database showed the effectiveness of our method for cross-view gait recognition.

\section{Appendix A. Derivation rewriting Eq. (5) into Eq. (6)}

$\mathbf{S}_{x y}$ is a matrix of which each element is $s_{i j}^{x y}$. Each element of $\mathbf{S}_{\mathbf{x}}$ and $\mathbf{S}_{\mathbf{y}}$ is $s_{i k}^{x}$ and $s_{j l}^{y}$ respectively. $\mathbf{D}_{\mathbf{x y}}^{\mathbf{c}}$ and $\mathbf{D}_{\mathbf{x y}}^{\mathbf{r}}$ are diagonal matrices; their entries are column and row sums of $\mathbf{S}_{x y} . \mathbf{D}_{\mathbf{x}}$ and $\mathbf{D}_{\mathbf{y}}$ are diagonal matrices; their entries are column (or row) sums of $\mathbf{S}_{x}$ and $\mathbf{S}_{y}$ respectively. Let $\mathbf{Z}=\left[\begin{array}{cc}\mathbf{X} & \\ & \mathbf{Y}\end{array}\right], \mathbf{W}=\left[\begin{array}{l}\mathbf{W}_{x} \\ \mathbf{W}_{y}\end{array}\right], \mathbf{S}=\left[\begin{array}{cc}\mathbf{S}_{x} & \mathbf{S}_{x y} \\ \mathbf{S}_{x y}^{T} & \mathbf{S}_{y}\end{array}\right]$, 


$$
\begin{aligned}
& \mathbf{D}=\left[\begin{array}{cc}
\mathbf{D}_{x}+\mathbf{D}_{x y}^{r} & \\
& \mathbf{D}_{y}+\mathbf{D}_{x y}^{c}
\end{array}\right] \text { and } \mathbf{L}=\mathbf{D}-\mathbf{S}, \text { then the derivation process of our objective function is as following. } \\
& J\left(\mathbf{W}_{x}, \mathbf{W}_{y}\right) \\
& =\sum_{i=1}^{m_{x}} \sum_{j=1}^{m_{y}}\left(\mathbf{W}_{x}^{T} \mathbf{x}_{i}-\mathbf{W}_{y}^{T} \mathbf{y}_{j}\right)^{2} s_{i j}^{x y}+\frac{1}{2} \sum_{i=1}^{m_{x}} \sum_{k=1}^{m_{x}}\left(\mathbf{W}_{x}^{T} \mathbf{x}_{i}-\mathbf{W}_{x}^{T} \mathbf{x}_{k}\right)^{2} s_{i k}^{x}+\frac{1}{2} \sum_{j=1}^{m_{y}} \sum_{l=1}^{m_{y}}\left(\mathbf{W}_{y}^{T} \mathbf{y}_{l}-\mathbf{W}_{y}^{T} \mathbf{y}_{j}\right)^{2} s_{j l}^{y} \\
& \left.=\operatorname{tr}\left(\mathbf{W}_{x}^{T} \mathbf{X} \mathbf{D}_{x y}^{r} \mathbf{X}^{T} \mathbf{W}_{x}+\mathbf{W}_{y}^{T} \mathbf{Y} \mathbf{D}_{x y}^{c} \mathbf{Y}^{T} \mathbf{W}_{y}-\mathbf{W}_{x}^{T} \mathbf{X} \mathbf{S}_{x y} \mathbf{Y}^{T} \mathbf{W}_{y}-\mathbf{W}_{y}^{T} \mathbf{Y} \mathbf{S}_{x y}^{T} \mathbf{X}^{T} \mathbf{W}_{x}\right)\right\} \\
& +\frac{1}{2}\left\{2 \times \operatorname{tr}\left[\mathbf{W}_{x}^{T} \mathbf{X}\left(\mathbf{D}_{x}-\mathbf{S}_{x}\right) \mathbf{X}^{T} \mathbf{W}_{x}\right]+2 \times \operatorname{tr}\left[\mathbf{W}_{y}^{T} \mathbf{Y}\left(\mathbf{D}_{y}-\mathbf{S}_{y}\right) \mathbf{Y}^{T} \mathbf{W}_{y}\right]\right. \\
& =\operatorname{tr}\left\{\mathbf{W}_{x}^{T} \mathbf{X}\left(\mathbf{D}_{x}+\mathbf{D}_{x y}^{r}-\mathbf{S}_{x}\right) \mathbf{X}^{T} \mathbf{W}_{x}+\mathbf{W}_{y}^{T} \mathbf{Y}\left(\mathbf{D}_{y}+\mathbf{D}_{x y}^{c}-\mathbf{S}_{y}\right) \mathbf{Y}^{T} \mathbf{W}_{y}-\mathbf{W}_{x}^{T} \mathbf{X} \mathbf{S}_{x y} \mathbf{Y}^{T} \mathbf{W}_{y}-\mathbf{W}_{y}^{T} \mathbf{Y} \mathbf{S}_{x y}^{T} \mathbf{X}^{T} \mathbf{W}_{x}\right\} \\
& =\operatorname{tr}\left\{\left[\begin{array}{ll}
\mathbf{W}_{x}^{T} \mathbf{X} & \mathbf{W}_{y}^{T} \mathbf{Y}
\end{array}\right]\left[\begin{array}{cc}
\mathbf{D}_{x}+\mathbf{D}_{x y}^{r} & \\
& \mathbf{D}_{y}+\mathbf{D}_{x y}^{c}
\end{array}\right]\left[\begin{array}{c}
\mathbf{X}^{T} \mathbf{W}_{x} \\
\mathbf{Y}^{T} \mathbf{W}_{y}
\end{array}\right]-\left[\begin{array}{ll}
\mathbf{W}_{x}^{T} \mathbf{X} & \mathbf{W}_{y}^{T} \mathbf{Y}
\end{array}\right]\left[\begin{array}{cc}
\mathbf{S}_{x} & \mathbf{S}_{x y} \\
\mathbf{S}_{x y}^{T} & \mathbf{S}_{y}
\end{array}\right]\left[\begin{array}{c}
\mathbf{X}^{T} \mathbf{W}_{x} \\
\mathbf{Y}^{T} \mathbf{W}_{y}
\end{array}\right]\right\} \\
& =\operatorname{tr}\left\{\left[\begin{array}{l}
\mathbf{W}_{x} \\
\mathbf{W}_{y}
\end{array}\right]^{T}\left[\begin{array}{cc}
\mathbf{X} & \\
& \mathbf{Y}
\end{array}\right](\mathbf{D}-\mathbf{S})\left[\begin{array}{ll}
\mathbf{X} & \\
& \mathbf{Y}
\end{array}\right]^{T}\left[\begin{array}{l}
\mathbf{W}_{x} \\
\mathbf{W}_{y}
\end{array}\right]\right\} \\
& =\operatorname{tr}\left\{\mathbf{W}^{T} \mathbf{Z}(\mathbf{D}-\mathbf{S}) \mathbf{Z}^{T} \mathbf{W}\right\} \\
& =\operatorname{tr}\left\{\mathbf{W}^{T} \mathbf{Z L Z} \mathbf{Z}^{T} \mathbf{W}\right\}
\end{aligned}
$$

\section{Acknowledgements}

This project is supported by the National Science Foundation of China (Grant No. 61071214), the Natural Science Foundation of the Higher Education Institutions of Jiangsu Province (Grant No. 16KJB110022), and the Funding of Jiangsu Innovation Program for Graduate Education (Grant No. KYZZ_0340).

\section{References}

[1] S Sarkar, P. J. Phillips, Z. Liu, I. R. Vega, P Grother, and K. W. Bowyer. The humanid gait challenge problem: data sets, performance, and analysis. IEEE Transactions on Pattern Analysis and Machine Intelligence, 27(2):162-77, 2005.

[2] Shiqi Yu, Daoliang Tan, and Tieniu Tan. A framework for evaluating the effect of view angle, clothing and carrying condition on gait recognition. In Proceedings 18th International Conference on Pattern Recognition, pages 441-444, 2006.

[3] R. Bodor, A. Drenner, D. Fehr, O. Masoud, and N. Papanikolopoulos. View-independent human motion classification using image-based reconstruction. Image and Vision Computing, 27(8):1194-1206, 2009.

[4] G. Shakhnarovich, L. Lee, and T. Darrell. Integrated face and gait recognition from multiple views. In Computer Vision and Pattern Recognition, Proceedings of the 2001 IEEE Computer Society Conference on, pages 439-446 vol.1, 2001.

[5] R. D. Seely, S. Samangooei, M. Lee, J. N. Carter, and M. S. Nixon. The university of southampton multi-biometric tunnel and introducing a novel 3d gait dataset. In IEEE International Conference on Biometrics: Theory, Applications and Systems, pages 1-6, 2008.

[6] Frdric Jean, Robert Bergevin, and Alexandra Branzan Albu. Computing and evaluating view-normalized body part trajectories. Image and Vision Computing, 27(9):1272-1284, 2009.

[7] Amit Kale, Amit K. Roy Chowdhury, and Rama Chellappa. Towards a view invariant gait recognition algorithm. In IEEE Conference on Advanced Video and Signal Based Surveillance, pages 143-150, 2003.

[8] M Goffredo, I Bouchrika, J. N. Carter, and M. S. Nixon. Self-calibrating view-invariant gait biometrics. IEEE Transactions on Systems Man and Cybernetics Part B, 40(4):997-1008, 2010.

[9] Shuai Zheng, Junge Zhang, Kaiqi Huang, Ran He, and Tieniu Tan. Robust view transformation model for gait recognition. In Proceedings International Conference on Image Processing, pages 2073-2076, 2011.

[10] Worapan Kusakunniran, Qiang Wu, Jian Zhang, and Hongdong Li. Support vector regression for multi-view gait recognition based on local motion feature selection. In IEEE Conference on Computer Vision and Pattern Recognition, pages 974-981, 2010.

[11] W. Kusakunniran, Qiang Wu, Jian Zhang, and Hongdong Li. Gait recognition under various viewing angles based on correlated motion regression. IEEE Transactions on Circuits and Systems for Video Technology, 22(6):966-980, 2012.

[12] Khalid Bashir, Tao Xiang, Shaogang Gong, Khalid Bashir, and Tao Xiang. Cross view gait recognition using correlation strength. Bmvc, pages $1-11,2010$.

[13] Bo Li, Hong Chang, Shiguang Shan, and Xilin Chen. Coupled metric learning for face recognition with degraded images. In Advances in Machine Learning, First Asian Conference on Machine Learning, ACML 2009, Nanjing, China, November 2-4, 2009. Proceedings, page 220, 2009.

[14] Kejun Wang, Xianglei Xing, Tao Yan, and Zhuowen Lv. Couple metric learning based on separable criteria with its application in cross-view gait recognition. Lecture Notes in Computer Science, 8833:347-356, 2014.

[15] Guoying Zhao, Guoyi Liu, Hua Li, and M. Pietikainen. 3d gait recognition using multiple cameras. In International Conference on Automatic Face and Gesture Recognition, pages 529-534, 2006.

[16] Yumi Iwashita, Ryosuke Baba, Koichi Ogawara, and Ryo Kurazume. Person identification from spatio-temporal 3d gait. In International Conference on Emerging Security Technologies, pages 30 - 35, 2010.

[17] W. Kusakunniran, Qiang Wu, Jian Zhang, Yi Ma, and Hongdong Li. A new view-invariant feature for cross-view gait recognition. IEEE Transactions on Information Forensics and Security, 8(10):1642-1653, 2013. 
[18] Yasushi Makihara, Ryusuke Sagawa, Yasuhiro Mukaigawa, Tomio Echigo, and Yasushi Yagi. Gait Recognition Using a View Transformation Model in the Frequency Domain. 2006.

[19] Daigo Muramatsu, Akira Shiraishi, Yasushi Makihara, Md Zasim Uddin, and Yasushi Yagi. Gait-based person recognition using arbitrary view transformation model. IEEE Transactions on Image Processing A Publication of the IEEE Signal Processing Society, 24(1):140-154, 2014.

[20] W. Kusakunniran, Qiang Wu, Jian Zhang, Hongdong Li, and Liang Wang. Recognizing gaits across views through correlated motion coclustering. IEEE Transactions on Image Processing A Publication of the IEEE Signal Processing Society, 23(2):696-709, 2014.

[21] Xianye Ben, Weixiao Meng, Rui Yan, and Kejun Wang. An improved biometrics technique based on metric learning approach. Neurocomputing, 97(1):44-51, 2012.

[22] Maodi Hu, Yunhong Wang, Zhaoxiang Zhang, J. J. Little, and Di Huang. View-invariant discriminative projection for multi-view gait-based human identification. IEEE Transactions on Information Forensics and Security, 8(12):2034-2045, 2013.

[23] Haifeng Hu. Multiview gait recognition based on patch distribution features and uncorrelated multilinear sparse local discriminant canonical correlation analysis. IEEE Transactions on Circuits and Systems for Video Technology, 24(4):617-630, 2014.

[24] Xianglei Xing, Kejun Wang, Tao Yan, and Zhuowen Lv. Complete canonical correlation analysis with application to multi-view gait recognition. Pattern Recognition, 50(C):107-117, 2015

[25] M Turk and A Pentland. Eigenfaces for recognition. Journal of Cognitive Neuroscience, 3(1):71-86, 1991.

[26] P N Belhumeur, J P Hespanha, and D J Kriegman. Eigenfaces vs. fisherfaces: Recognition using class specific linear projection. In IEEE Transactions on Pattern Analysis and Machine Intelligence, pages 711-720, 1997.

[27] Xiaofei He, Shuicheng Yan, Yuxiao Hu, Partha Niyogi, and Hong Jiang Zhang. Face recognition using laplacianfaces. IEEE Transactions on Pattern Analysis and Machine Intelligence, 27(3):328-340, 2005.

[28] J. B. Tenenbaum and W. T. Freeman. Separating style and content with bilinear models. Neural Computation, 12(6):1247-1283, 1989.

[29] Annan Li, Shiguang Shan, Xilin Chen, and Wen Gao. Face recognition based on non-corresponding region matching. pages 1060-1067, 2011.

[30] Yong Luo, Dacheng Tao, Yonggang Wen, Ramamohanarao Kotagiri, and Chao Xu. Tensor canonical correlation analysis for multi-view dimension reduction. Knowledge and Data Engineering IEEE Transactions on, 27(11):3111-3124, 2015.

[31] Abhishek Sharma and David W. Jacobs. Bypassing synthesis: Pls for face recognition with pose, low-resolution and sketch. In 2013 IEEE Conference on Computer Vision and Pattern Recognition, pages 593-600, 2011.

[32] L. An, M. Kafai, S. Yang, and B. Bhanu. Person re-identification with reference descriptor. IEEE Transactions on Circuits and Systems for Video Technology, pages 1-1, 2015.

[33] D. W. Jacobs, H. Daume, A. Kumar, and A. Sharma. Generalized multiview analysis: A discriminative latent space. In IEEE Conference on Computer Vision and Pattern Recognition, pages 2160-2167, 2012.

[34] Galen Andrew, Raman Arora, Jeff Bilmes, and Karen Livescu. Deep canonical correlation analysis. In ICML, pages 1247-1255, 2013.

[35] Wen Wang, Zhen Cui, Hong Chang, Shiguang Shan, and Xilin Chen. Deeply coupled auto-encoder networks for cross-view classification. Computer Science, 2014

[36] J. Han and Bhanu. B.: Individual recognition using gait energy image. IEEE Transation on Pattern Analysis and Machine Intelligence, $28(2): 316-322,2006$ 